PHYSICAL REVIEW A 87, 043403 (2013)

\title{
Torsional effects in molecular alignment
}

\author{
Juan Ortigoso* \\ Instituto de Estructura de la Materia, CSIC, Serrano 121, 28006 Madrid, Spain
}

\begin{abstract}
L. H. Coudert ${ }^{\dagger}$
Laboratoire Inter-universitaire des Systèmes Atmosphériques, UMR 7583 du CNRS, Universités Paris Est Créteil et Paris Diderot, 61 Avenue du Général de Gaulle, 94010 Créteil Cedex, France
\end{abstract}

(Received 20 November 2012; published 5 April 2013)

\begin{abstract}
A detailed theoretical formalism for the calculation of energy levels and eigenfunctions of molecules with a large amplitude coordinate in the presence of a strong laser pulse is developed based on a discrete variable representation to setup the Hamiltonian matrix. This approach is applied to nonrigid biphenyl-like molecules displaying a large amplitude motion corresponding to respective rotations of their two groups. The eigenvalues and eigenvectors obtained in several limiting cases of the hindering potential can be symmetry labeled and provide us with useful insights into the feasibility of torsional alignment. The present results support the rotation-induced breakdown of torsional alignment, under adiabatic following, previously described by Coudert, Pacios, and Ortigoso [Phys. Rev. Lett. 107, 113004 (2011)].
\end{abstract}

DOI: 10.1103/PhysRevA.87.043403

PACS number(s): $33.57 .+\mathrm{c}, 33.15 . \mathrm{Bh}$

\section{INTRODUCTION}

The behavior of molecules subject to intense laser fields has been intensively studied in recent years in order to investigate the formation of oriented or aligned states, quantum control, and solutions of the time-dependent Schrödinger equation. In some of these investigations, for instance, those reported in Refs. [1-5], the molecules were assumed to be rigid and were treated as rigid rotators undergoing overall rotation only. In more recent investigations, internal degrees of freedom were taken into account. Vibrational degrees of freedom were considered in Ref. [6] and a large amplitude internal rotation was treated in Refs. [7-12]. Guidelines for the realization of torsional control experiments have been provided by Seideman and coworkers [13], who proposed applications of torsional control to several problems like electronic transport in molecular junctions or charge transfer events. The theoretical approach of Ref. [13] is based on the quantum Liouville equation that allows them to take into account dissipation. However, these investigations [6-13] were performed ignoring the overall rotation of the molecule. Recently, thanks to a new approach rigorously taking into account the large amplitude torsional motion and the overall rotation, results about the behavior of a nonrigid biphenyl-like molecule subject to an intense nonresonant laser field were reported [14].

In the present paper, we give a full account of the theoretical approach introduced in Ref. [14]. Our approach treats simultaneously the large amplitude torsional motion and the overall rotation and involves using a discrete variable representation (DVR) similar to that introduced in Refs. [15,16] to solve a multidimensional time-independent Schrödinger equation. The method is applied to nonrigid biphenyl-like molecules undergoing internal rotation of their two groups and displaying a torsionally mediated interaction with an external electric

\footnotetext{
*j.ortigoso@csic.es

†laurent.coudert@lisa.u-pec.fr
}

field. The theoretical approach allows us to retrieve energies and wave functions and to label them with their symmetry species [17] in $G_{16}^{(2)}$ for all strengths of the electric field. As a test case, numerical results are computed for the biphenyl molecule. Specifically, expectation values of several rovibrational operators are evaluated for selected rotationtorsion-Stark levels, for various strengths of the electric field, and for various model hindering potentials. Thermal averages are also calculated for different temperatures.

The paper has four remaining sections. In Sec. II, the new theoretical approach is presented. It is applied to the particular case of biphenyl-like molecules in Sec. III. In Sec. IV, numerical results are presented about the biphenyl molecule. Section V is the discussion.

\section{THEORY}

Four coordinates are necessary to describe a nonrigid molecule undergoing internal rotation: the usual Eulerian-type angles $\chi, \theta, \phi$, denoted $\Omega$, and the angle corresponding to the internal rotation, denoted $\rho$, with $0 \leqslant \rho \leqslant 2 \pi$. Using a molecule-fixed axis system with its origin located at the molecular center of mass, the rotation-torsion-Stark interaction Hamiltonian $H$ can be obtained using Eq. [9] of Ref. [18]:

$$
H=\frac{1}{2} \sum_{\gamma, \delta} J_{\gamma} \mu_{\gamma, \delta}(\rho) J_{\delta}+V(\rho)+H_{S}(\rho, \Omega),
$$

where $\gamma, \delta=x, y, z$, and $\rho ; J_{x}, J_{y}$, and $J_{z}$ are the components of the angular momentum in the molecule-fixed axis system; $J_{\rho}=-i \partial / \partial \rho$ is the momentum conjugated to $\rho ; \mu_{\gamma, \delta}(\rho)$ are the components of the inverse of the $4 \times 4$ generalized inertia tensor; $V(\rho)$ is the potential energy function; and $H_{S}(\rho, \Omega)$ describes the Stark coupling with the electric field. In Eq. (1), the potential energy function contains the mass-dependent pseudopotential energy given in Eq. [10] of Ref. [18]. The volume element to be used for the Hamiltonian in Eq. (1) is $d \rho \sin \theta d \theta d \phi d \chi$. 


\section{A. The rotation-torsion-Stark Hamiltonian in discrete variable representation}

In internal rotation problems, the wave function is usually expanded using as a finite basis representation (FBR) the freeinternal-rotation functions:

$$
f_{m}(\rho)=e^{i m \rho} / \sqrt{2 \pi},
$$

where $m$ is an integer such that $-N \leqslant m \leqslant+N$. These $2 N+$ 1 functions fulfill the orthogonality relation:

$$
\int_{0}^{2 \pi} f_{m}^{*}(\rho) f_{n}(\rho) d \rho=\delta_{m, n} .
$$

A weight function $\omega(\rho)$, as defined in Eq. (1) of Ref. [19], does not appear in this equation as it reduces to unity with the FBR functions of Eq. (2). In agreement with Refs. [15,16], the DVR functions $u_{\alpha}(\rho)$, where $\alpha$ is an integer such that $1 \leqslant \alpha \leqslant$ $2 N+1$, can be expressed in terms of FBR functions using the orthogonal transformation matrix $\mathbf{T}$ defined in agreement with Ref. [19] as

$$
T_{\alpha, m}=\sqrt{\omega_{\alpha}} f_{m}\left(\rho_{\alpha}\right),
$$

where $\rho_{\alpha}$ and $\omega_{\alpha}$ are the points and weights, respectively, of the $(2 N+1)$-Gauss quadrature. In the case of the FBR functions in Eq. (2), points and weights can be found in Eq. (A1) of the Appendix.

In the DVR, the matrix of the rotation-torsion-Stark interaction Hamiltonian in Eq. (1) is divided into $(2 N+$ $1) \times(2 N+1)$ submatrices. Each submatrix corresponds to a rotational operator, denoted $H^{\alpha, \alpha^{\prime}}$. This rotational operator is the following:

$$
\begin{aligned}
H^{\alpha, \alpha^{\prime}}= & \frac{1}{2} h^{\alpha, \alpha^{\prime}}+\delta_{\alpha, \alpha^{\prime}}\left[H_{r}\left(\rho_{\alpha}\right)+V\left(\rho_{\alpha}\right)\right] \\
& +\frac{1}{2} c^{\alpha, \alpha^{\prime}} \sum_{\gamma=x y z} J_{\gamma}\left[\mu_{\gamma, \rho}\left(\rho_{\alpha}\right)+\mu_{\gamma, \rho}\left(\rho_{\alpha^{\prime}}\right)\right],
\end{aligned}
$$

where $h^{\alpha, \alpha^{\prime}}$ and $c^{\alpha, \alpha^{\prime}}$ are matrix elements of $P_{\rho} \mu_{\rho, \rho}(\rho) P_{\rho}$ and $P_{\rho}$, respectively, between two DVR functions; and $H_{r}(\rho)$ is a rotational operator, which does not involve $P_{\rho}$. We have

$$
\begin{gathered}
c^{\alpha, \alpha^{\prime}}=\left\langle u_{\alpha}\left|P_{\rho}\right| u_{\alpha^{\prime}}\right\rangle, \\
h^{\alpha, \alpha^{\prime}}=\left\langle u_{\alpha}\left|P_{\rho} \mu_{\rho, \rho}(\rho) P_{\rho}\right| u_{\alpha^{\prime}}\right\rangle,
\end{gathered}
$$

and

$$
H_{r}(\rho)=\frac{1}{2} \sum_{\gamma, \delta=x y z} J_{\gamma} \mu_{\gamma, \delta}(\rho) J_{\delta}+H_{S}(\rho, \Omega) .
$$

Using Eq. (6) of Ref. [19], the matrix element $c^{\alpha, \alpha^{\prime}}$ in Eq. (6) can be expressed as

$$
c^{\alpha, \alpha^{\prime}}=\sum_{m=-N}^{+N} T_{\alpha, m} T_{\alpha^{\prime}, m}^{*} m .
$$

This equation ensures that $c^{\alpha, \alpha^{\prime}}=\left(c^{\alpha^{\prime}, \alpha}\right)^{*}$. Similarly, the matrix element $h^{\alpha, \alpha^{\prime}}$ in Eq. (7) can be written as

$$
h^{\alpha, \alpha^{\prime}}=\sum_{\alpha^{\prime \prime}=1}^{2 N+1} c^{\alpha, \alpha^{\prime \prime}} \mu_{\rho, \rho}\left(\rho_{\alpha^{\prime \prime}}\right) c^{\alpha^{\prime \prime}, \alpha^{\prime}} .
$$

This equation is consistent with $h^{\alpha, \alpha^{\prime}}=\left(h^{\alpha^{\prime}, \alpha}\right)^{*}$. When $\mu_{\rho, \rho}$ does not depend on the $\rho$ coordinate, Eq. (10) reduces to

$$
h^{\alpha, \alpha^{\prime}}=\mu_{\rho, \rho} \sum_{m=-N}^{+N} T_{\alpha, m} T_{\alpha^{\prime}, m}^{*} m^{2} .
$$

\section{B. Rotation-torsion-Stark eigenvalues and eigenfunctions}

The rotational operator $H_{r}(\rho)$ in Eq. (8) is the rotation-Stark coupling Hamiltonian of the molecule for a given value of $\rho$. This operator involves the overall rotation Hamiltonian and the Stark field interaction operator. Using Refs. [20,21], the matrix of $H_{r}(\rho)$ can be setup in a basis set consisting of symmetric top functions $|J, k, M\rangle$, where $J$ corresponds to the total angular momentum, $k$ to the eigenvalue of its projection along the molecule-fixed $z$ axis $J_{z}$, and $M$ to the eigenvalue of its projection along the space-fixed $Z$ axis $J_{Z}$. Taking a maximum $J$ value equal to $J_{\max }$ leads to a finite $p \times p$ matrix, where $p$ depends on $J_{\max }$ and on the rotational functions selected. Diagonalization of this matrix yields eigenvalues and eigenvectors depending implicitly on $\rho$ and denoted $E_{i}$ and $\psi_{i}$, where $i$ is a counter with $1 \leqslant i \leqslant p$. Below, $E_{i}^{\alpha}$ and $\psi_{i}^{\alpha}$ indicate eigenvalues and eigenvectors for $\rho=\rho_{\alpha}$.

The product functions $u_{\alpha}(\rho) \psi_{i}^{\alpha}$ are used to setup the matrix of the rotation-torsion-Stark Hamiltonian of Eq. (1). Using Eq. (5), we have

$$
\begin{aligned}
& \left\langle u_{\alpha}\right|\left\langle\psi_{i}^{\alpha}|H| \psi_{i^{\prime}}^{\alpha^{\prime}}|| u_{\alpha^{\prime}}\right\rangle \\
& =\frac{1}{2} h^{\alpha, \alpha^{\prime}}\left\langle\psi_{i}^{\alpha} \mid \psi_{i^{\prime}}^{\alpha^{\prime}}\right\rangle+\frac{1}{2} c^{\alpha, \alpha^{\prime}} \sum_{\gamma=x y z}\left\langle\psi_{i}^{\alpha}\left|J_{\gamma}\right| \psi_{i^{\prime}}^{\alpha^{\prime}}\right\rangle\left[\mu_{\gamma, \rho}\left(\rho_{\alpha}\right)\right. \\
& \left.\quad+\mu_{\gamma, \rho}\left(\rho_{\alpha^{\prime}}\right)\right]+\delta_{\alpha, \alpha^{\prime}} \delta_{i, i^{\prime}}\left[E_{i}^{\alpha}+V\left(\rho_{\alpha}\right)\right] .
\end{aligned}
$$

This equation emphasizes that the Hamiltonian matrix becomes a $\left[(2 N+1) i_{\max }\right] \times\left[(2 N+1) i_{\max }\right]$ matrix, where $i_{\max }$, with $i_{\max } \leqslant p$, is the number of rotational eigenvectors selected. Numerical diagonalization of this Hamiltonian matrix requires choosing for $N, J_{\max }$, and $i_{\max }$ values providing the needed accuracy.

\section{ROTATION-TORSION-STARK ENERGY LEVELS OF BIPHENYL-LIKE MOLECULES}

The approach of the previous section is applied to biphenyllike molecules consisting of two identical planar groups with $C_{2 v}$ symmetry. The large amplitude internal rotation displayed by such molecules correspond to a rotation of both groups about a fixed axis coinciding with their $C_{2}$ axes. In the case of the biphenyl molecule, the two groups are phenyl rings and the axis of internal rotation is the $\mathrm{C}-\mathrm{C}$ bond. The theoretical results derived by Merer and Watson [17] for ethylene can be used for biphenyl-like molecules. In agreement with these authors, the angle $\rho$ is such that $2 \rho$ is the angle of torsion of the two groups. The molecule-fixed axis system is attached to the molecule so that the $z$ axis is parallel to the axis of internal rotation; the $x$ and $y$ axis being parallel to two of the three twofold axes of symmetry. When $\rho=0$ or $\pi$, the molecule is planar and all atoms are in the $x z$ plane; when $\rho=\pi / 2$ or $3 \pi / 2$, the molecule is also planar, but all atoms are in the $y z$ plane. Equations (1) and (2) of Ref. [17] allow us to obtain 
nonvanishing components of the generalized inverse inertia tensor:

$$
\begin{aligned}
& \mu_{\rho, \rho}(\rho)=\mu_{z, z}(\rho)=2 A, \quad \mu_{x, x}(\rho)=2 B_{x}(\rho), \\
& \mu_{y, y}(\rho)=2 B_{y}(\rho),
\end{aligned}
$$

where $A$ is a structural parameter; and the rotational constants $B_{x}(\rho)$ and $B_{y}(\rho)$ should be obtained using Eq. (3) of Ref. [17] involving the structural parameter $B$. This leads to the following kinetic energy operator:

$$
A P_{\rho}^{2}+A J_{z}^{2}+B_{x}(\rho) J_{x}^{2}+B_{y}(\rho) J_{y}{ }^{2} .
$$

In the case of the biphenyl molecule, $A$ and $B$ are equal to 0.095833 and $0.016942 \mathrm{~cm}^{-1}$, respectively, as obtained from the $a b$ initio calculation reported in Ref. [14].

Because biphenyl-like molecules have no permanent dipole, the field-matter interaction reduces to the interaction of the electric field with the induced dipole, described by the polarizability tensor. It will be assumed that this tensor is the sum of that of each subunit. This leads to the results displayed in Eqs. (1) of Ref. [14], which emphasize that the total polarizability tensor depends on $\rho$. The molecule is subject to the electric field arising from a nonresonant circularly polarized laser beam propagating along the $Z$ axis with nonvanishing laboratory-fixed components $E_{X}=E \cos \omega t / \sqrt{2}$ and $E_{Y}=E \sin \omega t / \sqrt{2}$, where $E$ is the magnitude of the electric field and $\omega$ its angular frequency. The operator $H_{S}(\rho, \Omega)$ describing the interaction with the electric field obtained after time averaging is

$$
\begin{aligned}
H_{S}(\rho, \Omega)= & \frac{E^{2}}{\sqrt{96}}\left(\alpha_{x}^{0}-\alpha_{y}^{0}\right)\left(D_{20}^{(2)}+D_{-20}^{(2)}\right) \cos 2 \rho \\
& +\frac{E^{2}}{12}\left(2 \alpha_{z}^{0}-\alpha_{x}^{0}-\alpha_{y}^{0}\right) D_{00}^{(2)} \\
& -\frac{E^{2}}{6}\left(\alpha_{z}^{0}+\alpha_{x}^{0}+\alpha_{y}^{0}\right),
\end{aligned}
$$

where functions involving the Eulerian-type angles have been expressed in terms of Wigner $D^{(J)}$ functions defined as in Eq. (15.27) of Wigner's book [22]; and $\alpha_{x}^{0}, \alpha_{y}^{0}$, and $\alpha_{z}^{0}$ are diagonal components of the polarizability tensor of each molecular group with its atoms in the $x z$ plane and its $C_{2}$ axis parallel to the $z$ axis. Using Table I of Ref. [14], numerical values of -3.6 and $+3.6 \AA^{3}$ are obtained for $\alpha_{y}^{0}-\alpha_{x}^{0}$ and $2 \alpha_{z}^{0}-\alpha_{x}^{0}-\alpha_{y}^{0}$, respectively, in the case of the biphenyl

TABLE I. The four types of rotational functions to be used when diagonalizing the rotational operator $H_{r}(\rho)$ of Eq. (8) in the case of biphenyl-like molecules. ${ }^{a}$

\begin{tabular}{lccc}
\hline \hline Type & Even $J$ & Odd $J$ & $p^{\text {b }}$ \\
\hline$A$ & $E^{+}$ & $E^{-}$ & 57 \\
$B$ & $E^{-}$ & $E^{+}$ & 56 \\
$C$ & $O^{+}$ & $O^{-}$ & 56 \\
$D$ & $O^{-}$ & $O^{+}$ & 56 \\
\hline \hline
\end{tabular}

$\overline{{ }^{a} \text { In the body of the table, } E^{+}, E^{-}, O^{+} \text {, and } O^{-} \text {are usual Wang-type }}$ linear combinations of symmetric top functions.

${ }^{\mathrm{b}}$ The matrix to be diagonalized is $p \times p$ (see Sec. II B). The values given are for $J_{\max }=14$ molecule. With these values and expressing the Wigner $D^{(J)}$ functions in Eq. (15) in terms of $\theta$ and $\chi$ shows that the interaction with the electric field in this equation displays eight equivalent minima. Due to the double-valued characteristic of the coordinate system used in Ref. [17], there arise only four configurations with values of $(\theta, \chi, \rho)$ equal to $(\pi / 2,0,0)$, $(\pi / 2, \pi / 2, \pi / 2),(\pi / 2,0, \pi)$, and $(\pi / 2, \pi / 2,3 \pi / 2)$.

Evaluation of the rotational matrix elements of the Stark field interaction operator in Eq. (15) can be performed using Eq. (1.45) of Ref. [23] rewritten below:

$$
\begin{aligned}
\left\langle J^{\prime} k^{\prime} M^{\prime}\left|D_{p q}^{(k)}\right| J^{\prime \prime} k^{\prime \prime} M^{\prime \prime}\right\rangle= & (-1)^{k^{\prime}+M^{\prime}} \sqrt{\left(2 J^{\prime}+1\right)\left(2 J^{\prime \prime}+1\right)} \\
& \times\left(\begin{array}{lll}
J^{\prime} & k & J^{\prime \prime} \\
-k^{\prime} & p & k^{\prime \prime}
\end{array}\right) \\
& \times\left(\begin{array}{lll}
J^{\prime} & k & J^{\prime \prime} \\
-M^{\prime} & q & M^{\prime \prime}
\end{array}\right)
\end{aligned}
$$

This equation emphasizes that, as in Eq. (15), the second subscript of the Wigner $D^{(J)}$ functions is zero, the Stark field interaction operator will have vanishing $\Delta M \neq 0$ matrix elements, and $M$ will be a good quantum number. Due to the form of the $|\Delta J|>0$ and $|\Delta k|>0$ matrix elements of the Stark field interaction operator, it can also be shown that four types of rotational functions should be considered when setting-up the matrix of the rotational operator $H_{r}(\rho)$ in Eq. (8). These four types are defined in Table I along with the size of the matrix $p$ for $J_{\max }=14$. Figures 1 and 2 illustrate the results of the calculation for the lowest lying eigenvalues and for two values of the intensity of the laser beam in the case of the biphenyl molecule.

We are led to consider FBR functions that are even or odd functions of $\rho$. The even functions, denoted $C_{n}(\rho)$, with $0 \leqslant$ $n \leqslant N$, are cosine functions: $C_{0}(\rho)=1 / \sqrt{2 \pi}$ and $C_{n}(\rho)=$ $\cos (n \rho) / \sqrt{\pi}$ for $n>0$. The odd functions, denoted $S_{n}(\rho)$, with $1 \leqslant n \leqslant N$, are sine functions: $S_{n}(\rho)=\sin (n \rho) / \sqrt{\pi}$. The weights and points for the $C_{n}(\rho)$ and $S_{n}(\rho)$ functions can be found in Eqs. (A4) and (A5), respectively. The Hamiltonian matrix is set-up using Eq. (12). Depending on the FBR

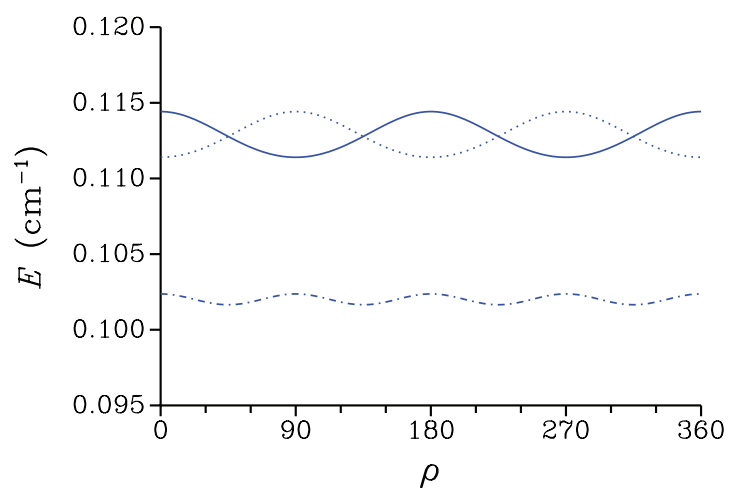

FIG. 1. (Color online) Variations of three rotational eigenvalues $E_{i}$ of the rotational Hamiltonian $H_{r}(\rho)$ of Eq. (8) for the biphenyl molecule as a function of the angle $\rho$ in degrees in the field-free limit. The dot-dashed curve is an $i=2$ eigenvalue corresponding to Type $A$ rotational functions. The solid (dotted) curve is an $i=1$ eigenvalue corresponding to Type $C(D)$. The three eigenvalues are periodic functions of $\rho$ with $\pi / 2$ or $\pi$ periodicity. 


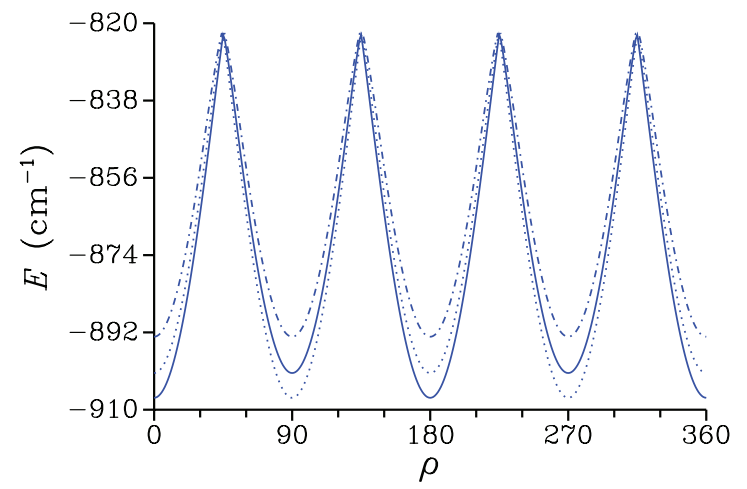

FIG. 2. (Color online) Variations of three $M=0$ rotational eigenvalues $E_{i}$ of the rotational Hamiltonian $H_{r}(\rho)$ as a function of the angle $\rho$ in degrees for a circularly polarized laser field with an intensity $I=1.562 \times 10^{12} \mathrm{~W} / \mathrm{cm}^{2}$. The $i$ values and the rotational types are the same as described in the legend of Fig. 1. The same periodicity as in this figure is displayed.

functions chosen and on the type of rotational functions, up to eight matrices can be built. Table II gives the symmetry species spanned by the rotation-torsion-Stark interaction levels arising for each matrix. This result can be obtained using Table IV of Ref. [17]. Each matrix is block diagonalized in order to obtain the eigenvalue and eigenvectors corresponding to one of the 12 single-valued symmetry species of $G_{16}^{(2)}$.

\section{NUMERICAL RESULTS}

The results of Sec. III are applied to the calculation of the rotation-torsion-Stark interaction energy levels of the biphenyl molecule considering four limiting cases for the internal rotation potential energy function $V(\rho)$. This function is assumed to be either zero (Case I); to have four minima at the eclipsed configurations (Case II); to have 4 minima at the staggered configurations (Case III); or to be the potential energy function obtained through ab initio calculations in Ref. [14] (Case IV).

TABLE II. The symmetry species of the rotation-torsion-Stark interaction levels.

\begin{tabular}{lcccc}
\hline \hline Function $^{\mathrm{a}}$ & Type $^{\mathrm{b}}$ & $\Gamma^{\mathrm{c}}$ & & \\
\hline$C_{n}(\rho)$ & $A$ & $A_{1 g}^{+}$ & $B_{1 g}^{+}$ & $E_{1}(u)$ \\
$C_{n}(\rho)$ & $B$ & $A_{2 g}^{-}$ & $B_{2 g}^{-}$ & $E_{2}(u)$ \\
$C_{n}(\rho)$ & $C$ & $E^{-}(1)$ & $E_{g}(1)$ & \\
$C_{n}(\rho)$ & $D$ & $E^{+}(2)$ & $E_{g}(2)$ & \\
$S_{n}(\rho)$ & $A$ & $A_{1 u}^{-}$ & $B_{1 u}^{-}$ & $E_{1}(g)$ \\
$S_{n}(\rho)$ & $B$ & $A_{2 u}^{+}$ & $B_{2 u}^{+}$ & $E_{2}(g)$ \\
$S_{n}(\rho)$ & $C$ & $E^{+}(1)$ & $E_{u}(1)$ & \\
$S_{n}(\rho)$ & $D$ & $E^{-}(2)$ & $E_{u}(2)$ & \\
\hline \hline
\end{tabular}

a the text.

${ }^{\mathrm{b}}$ The rotational function type is given in this column as defined in Table I.

${ }^{\mathrm{c}}$ The symmetry species spanned by the resulting rotation-torsionStark energy levels are given in this column using the same labeling as in Ref. [17].
TABLE III. Rotational assignment and energies are given for field-free $n=1$ rotation-torsion-Stark interaction levels ${ }^{\text {a }}$ for the four internal rotation potentials.

\begin{tabular}{|c|c|c|c|c|c|c|c|c|}
\hline \multirow[b]{3}{*}{$\Gamma$} & \multicolumn{8}{|c|}{ Case } \\
\hline & \multicolumn{2}{|r|}{ I } & \multicolumn{2}{|r|}{ II } & \multicolumn{2}{|r|}{ III } & \multicolumn{2}{|r|}{ IV } \\
\hline & $J$ & $E$ & $J$ & $E$ & $J$ & $E$ & $J$ & $E$ \\
\hline$A_{1 g}^{+}$ & 0 & 0.00 & 0 & 13.748 & 0 & 13.748 & 0 & 30.745 \\
\hline$B_{1 g}^{+}$ & 0 & 0.383 & 0 & 13.748 & 2 & 14.165 & 0 & 30.745 \\
\hline$A_{2 g}^{-}$ & 1 & 0.034 & 1 & 13.782 & 1 & 13.782 & 1 & 30.779 \\
\hline$B_{2 g}^{-}$ & 2 & 0.417 & 1 & 13.782 & 2 & 14.165 & 2 & 30.779 \\
\hline$A_{1 u}^{-}$ & 0 & 0.383 & 0 & 41.048 & 0 & 13.748 & 0 & 30.745 \\
\hline$B_{1 u}^{-}$ & 0 & 0.801 & 0 & 41.048 & 2 & 14.165 & 0 & 30.745 \\
\hline$A_{2 u}^{+}$ & 1 & 0.417 & 1 & 41.082 & 1 & 13.782 & 1 & 30.779 \\
\hline$B_{2 u}^{+}$ & 2 & 0.801 & 1 & 41.082 & 1 & 41.082 & 2 & 30.779 \\
\hline$E^{-}$ & 1 & 0.208 & 1 & 13.859 & 1 & 13.861 & 1 & 30.856 \\
\hline$E^{+}$ & 1 & 0.209 & 1 & 13.862 & 1 & 13.861 & 1 & 30.857 \\
\hline
\end{tabular}

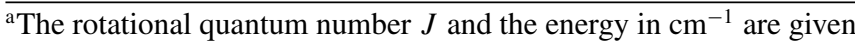
in the columns headed $J$ and $E$, respectively.

Cases I-III were considered in our previous paper [14], and analytical expressions for the potential energy function can be found in Table I of this reference. In Case IV, the analytical expression taken for the internal rotation potential energy function is

$$
V(\rho)=V_{0}-1 / 2 \sum_{i=1}^{6} V_{4 i} \cos 4 i \rho,
$$

where the constants $V_{4 i}$, with $i=1$ to 6 , are equal to -32.288 , $-738.683,-136.964,-84.92,-41.061$, and $-32.562 \mathrm{~cm}^{-1}$, and $V_{0}=345.34 \mathrm{~cm}^{-1}$. This value ensures that the potential energy function is 0 at the eight equivalent minima for values of $\rho$ equal to $22,68,112,158,202,248,292$, and $338^{\circ}$. The height of the barrier for the staggered and planar configurations are 670 and $860 \mathrm{~cm}^{-1}$, respectively.

Rotation-torsion-Stark interaction energies will be denoted $E_{M, n}^{\Gamma}$, where $n$, with $n \geqslant 1$, is a counter and $\Gamma$ is the symmetry species in $G_{16}^{(2)}$. For all cases, Table III gives calculated fieldfree energies for $n=1$ rotation-torsion-Stark interaction levels and for all ten single-valued symmetry species of $G_{16}^{(2)}$. In the field-free limit, levels can also be labeled [20] with the quantum number $J$, which is also given in Table III. In the next sections, for a given rotation-torsion-Stark interaction level, the expectation value of an operator $O$ is calculated as

$$
\langle O\rangle=\int \Psi_{M, n}^{\Gamma *} O \Psi_{M, n}^{\Gamma} d \Omega d \rho .
$$

In these numerical calculations, $J_{\max }$ in Sec. II B was taken equal to 14 and $i_{\max }$ defined in the same section was set to its maximum value, given in Table I. $N$ in Sec. II A was set to 91 and 93 for $g$-type (gerade) and $u$-type (ungerade) nondegenerate levels, respectively; and to 92 for doubly degenerate levels. These values ensure converged energy levels up to the maximum intensity value of the laser field, $2 \times 10^{14} \mathrm{~W} / \mathrm{cm}^{2}$. From the experimental point of view, this intensity value is above the threshold of off-resonance ionization of biphenyl, but this will be ignored in the present theoretical calculation. 


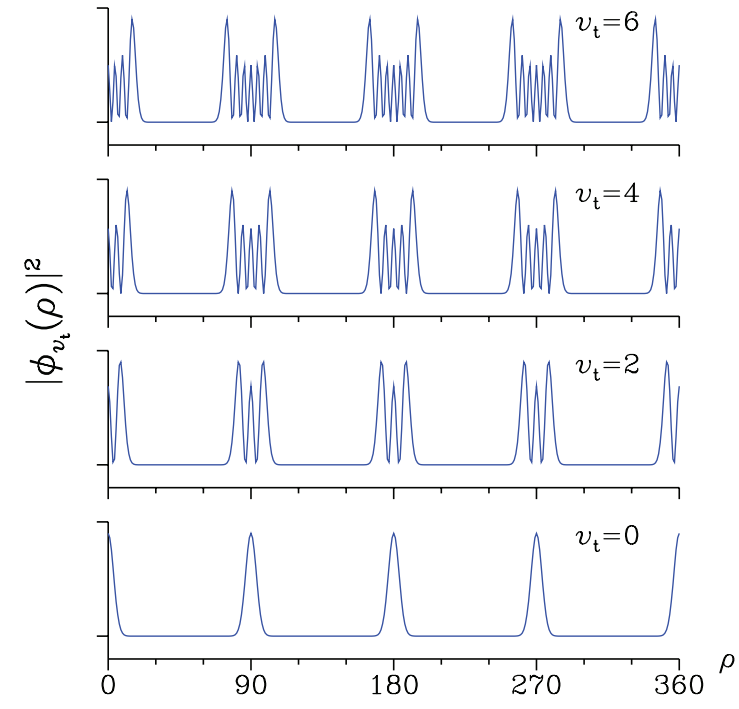

FIG. 3. (Color online) Variations with $\rho$ (in degrees) of the squared field-free torsional functions $\left|\phi_{v_{t}}(\rho)\right|^{2}$ for Case II potential and for $A_{1 g}^{+}$field-free levels with $J=0$.

Figure 3 shows the torsional function squared $\left|\phi_{v_{t}}(\rho)\right|^{2}$ for Case II potential and for field-free $A_{1 g}^{+}$levels with $J=0$. This figure serves the purpose of explaining some effects due to the electric field described below. These functions can be labeled using the torsional quantum number $v_{t}$ and display one or several maxima near the values of $\rho$ minimizing the hindering potential. Each maximum consists of $v_{t}+1$ close lying peaks.

\section{A. Averaged torsional function}

Taking $O=\delta\left(\rho-\rho_{0}\right)$ in Eq. (18) yields the averaged torsional function squared. Its variations were studied with the help of 2D surfaces plotted against the intensity of the laser beam $I$ and the angle $\rho_{0}$. Figure 4 shows the surface for Case I potential and for the $n=1, M=0, A_{1 g}^{+}$rotation-torsion-Stark interaction level, correlating to a $J=0$ level in the field-free limit. This figure emphasizes that for a low intensity value, the torsional function is a constant, as expected for an $0^{+}$ free internal rotation state [17]. When the intensity of the laser beam increases, the torsional function tends to become localized near values of $\rho_{0}$ minimizing the coupling with the electric field: $0,90,180,270$, and $360^{\circ}$. The surface for Case II potential, also for the same level, is not shown here because it is barely altered when the laser field is turned on. In the field-free limit, this function is localized near $\rho_{0}=0,90,180$, 270 , and $360^{\circ}$, due to the nature of the hindering potential. When the intensity of the laser field increases, the function remains localized near these values of $\rho_{0}$. Figure 5 shows the surface for Case III potential and for the same level. This figure clearly indicates that in the field-free limit the averaged function is localized near values of $\rho_{0}$ equal to $45,135,225$, and $315^{\circ}$, corresponding to the minima of the hindering potential. For the largest intensity value, the function is almost localized near $\rho_{0}=0,90,180,270$, and $360^{\circ}$. The surface for Case IV potential and for the same level, not shown here, is consistent with a torsional function localized near the eight values of $\rho_{0}$ minimizing the potential energy function in the field-free limit. When the intensity of the laser beam increases, only

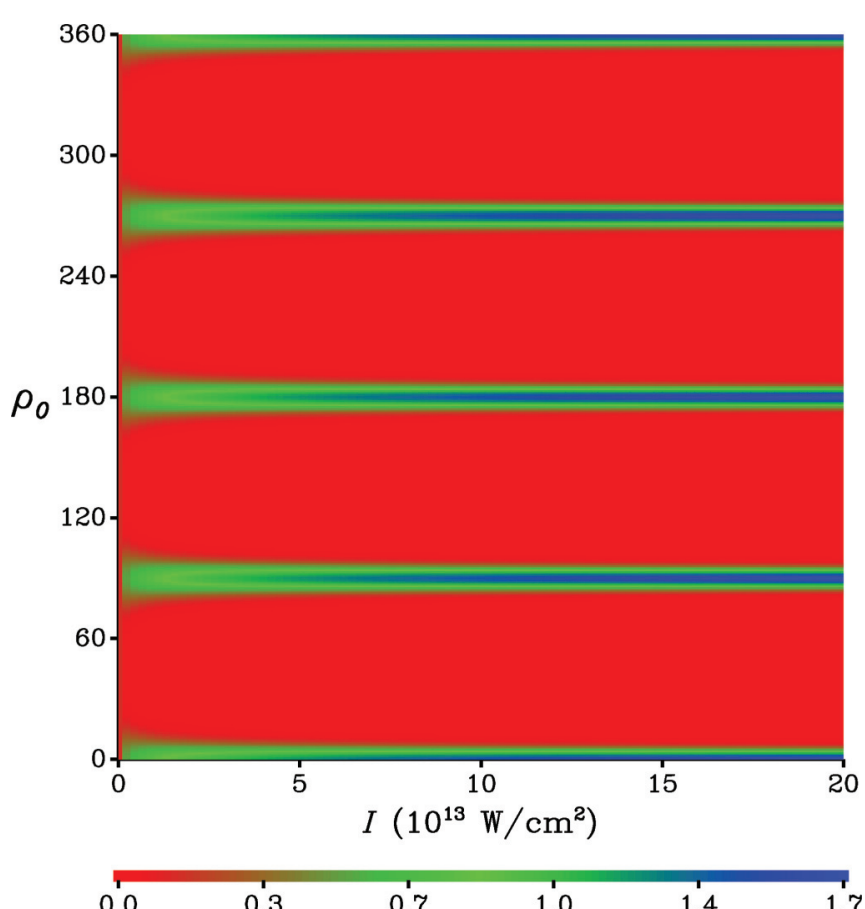

FIG. 4. (Color online) Variations of the averaged torsional function in Case I potential for the $n=1, M=0, A_{1 g}^{+}$, rotation-torsionStark interaction level correlating adiabatically to a $J=0$ level in the field-free limit.

slight shifts of the maxima of the function take place. Such a behavior is expected since the barriers between the various configurations are higher in Case IV than in Cases I, II, and III.

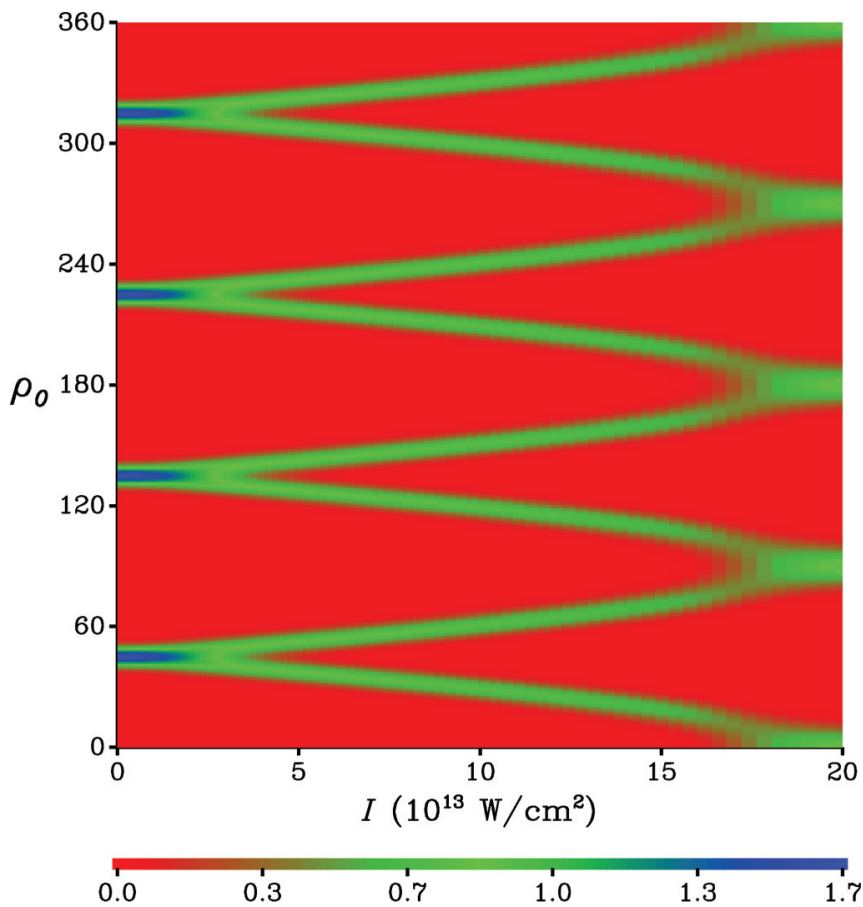

FIG. 5. (Color online) Variations of the averaged torsional function in Case III potential for the $n=1, M=0, A_{1 g}^{+}$, rotation-torsionStark interaction level correlating adiabatically to a $J=0$ level in the field-free limit. 


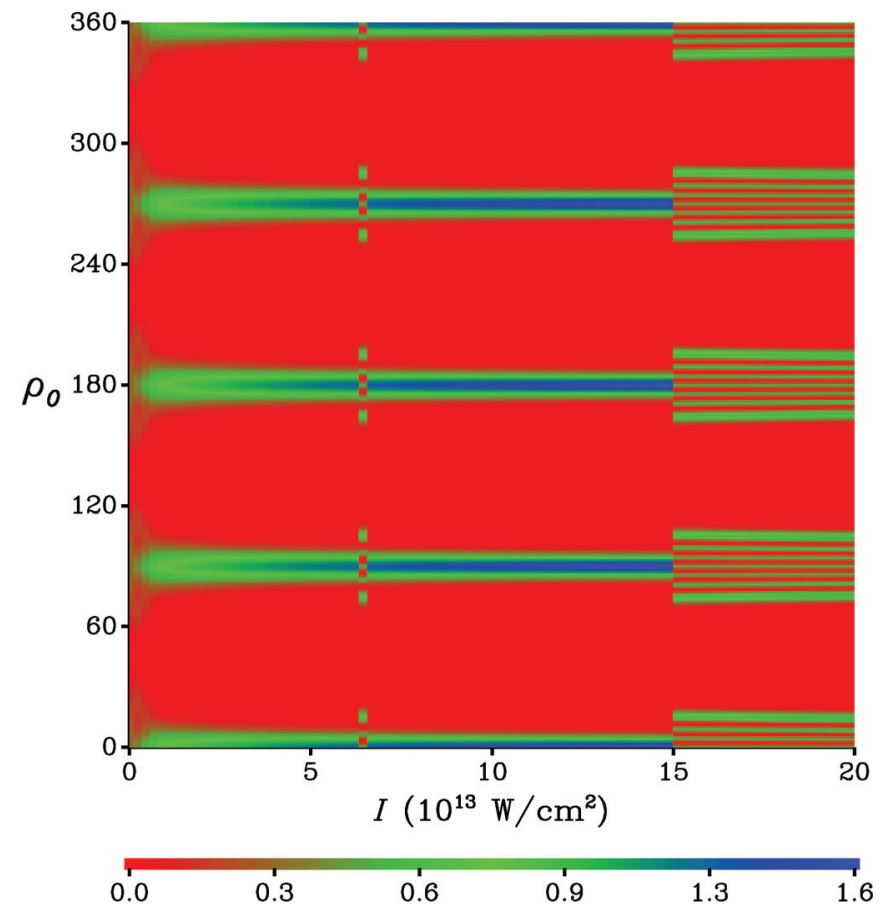

FIG. 6. (Color online) Variations of the averaged torsional function in Case I potential for the $n=5, M=0, A_{1 g}^{+}$, rotation-torsionStark interaction level correlating adiabatically to a $J=3$ level in the field-free limit.

In Case IV, the electric field cannot alter the torsional function and torsional alignment does not take place.

For levels correlating to higher $J$ values than in the previous cases, the effects of the electric field are more complicated. For Case I potential, Fig. 6 shows the torsional functions of an $n=5, M=0, A_{1 g}^{+}$level correlating to a $J=3$ level in the field-free limit. For low intensity values, the averaged torsional function displays four maxima as expected for a $2^{+}$ free internal rotation state [17]. For higher intensity values, the function tends to become localized near the values of $\rho_{0}$ minimizing the coupling with the electric field: 0, 90, 180, 270, and $360^{\circ}$. Unlike for the $n=1$ level dealt with previously, the variations of the function with the electric field are not smooth. For several values of the intensity, as well as for the largest one, the function displays broad maxima consisting of close lying peaks. Figure 7 shows similar results, but for Case II potential and for an $n=2, M=0, A_{1 g}^{+}$level correlating to a $J=2$ level in the field-free limit. When the intensity of the laser beam is zero, the averaged torsional function is localized near $\rho_{0}=0,90,180,270$, and $360^{\circ}$, due to the nature of the hindering potential. For small intensity values, the function remains localized near these values of $\rho_{0}$. For an intensity larger than $7.5 \times 10^{13} \mathrm{~W} / \mathrm{cm}^{2}$, the function displays several broad maxima consisting of close lying peaks near the same values of $\rho_{0}$. For Case III potential, the results are analogous to those of Cases I and II. For high intensity values, the function also displays broad minima consisting of several close lying peaks. For Case IV potential, the torsional function is quite close to that described in the previous paragraph and is barely altered by the electric field. For Cases I, II, and III, the qualitative features of the torsional function for the highest

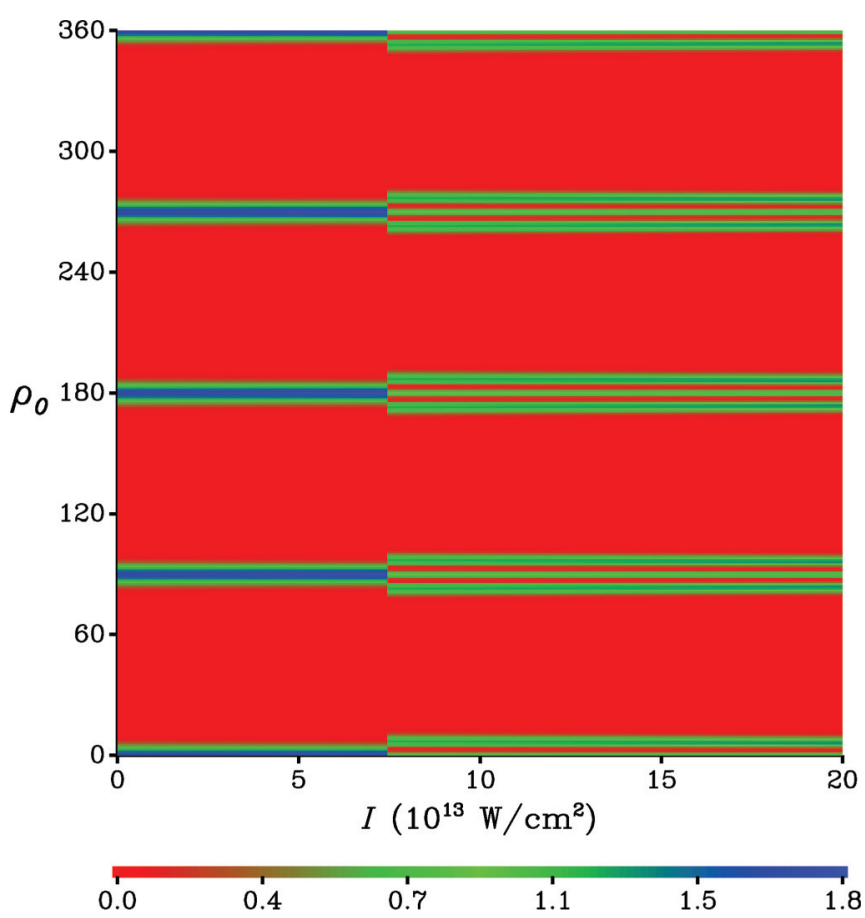

FIG. 7. (Color online) Variations of the averaged torsional function in Case II potential for the $n=2, M=0, A_{1 g}^{+}$, rotation-torsionStark interaction level correlating adiabatically to a $J=2$ level in the field-free limit.

value of the intensity of the laser beam may be unexpected. However, the functions in these cases resemble the functions plotted in Fig. 3 for large $v_{t}$ values. This similarity implies that due to the electric field, the torsional function for levels correlating to higher $J$ values in the field-free limit is mixed with that of levels characterized by large $v_{t}$ values.

\section{B. Expectation value of the torsional angle}

The expectation value of the torsional angle $\rho_{e}$ is calculated setting $O$ to $\cos 4 \rho$ in Eq. (18). The angle $\rho_{e}$ is then obtained from $\rho_{e}=\frac{1}{4} \cos ^{-1}\langle\cos 4 \rho\rangle$. Figure 8 shows the variations of $\rho_{e}$, for Case I potential, for $M=0, A_{1 g}^{+}$rotation-torsion-Stark

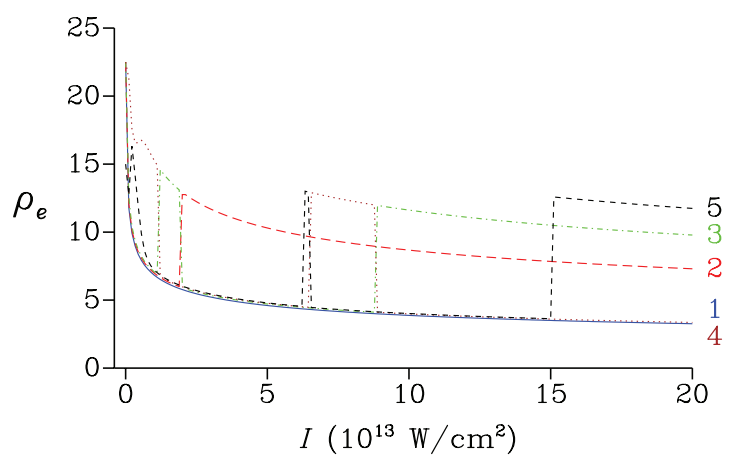

FIG. 8. (Color online) The expectation value of the torsional angle $\rho_{e}$ is plotted as a function of the laser beam intensity in Case I potential for $M=0, A_{1 g}^{+}$rotation-torsion-Stark interaction levels with $n=1$, solid line, $n=2$, long dashed line, $n=3$, dot-dashed line, $n=4$, dotted line, and $n=5$, dashed line. The value of $n$ is also indicated for each curve. 


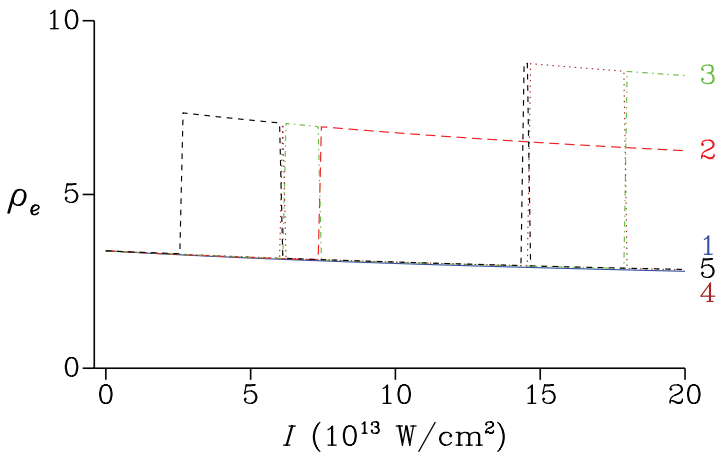

FIG. 9. (Color online) The expectation value of the torsional angle $\rho_{e}$ is plotted as a function of the laser beam intensity in Case II potential for $M=0, A_{1 g}^{+}$rotation-torsion-Stark interaction levels with $n=1$, solid line; $n=2$, long dashed line; $n=3$, dot-dashed line; $n=4$, dotted line; and $n=5$, dashed line. The value of $n$ is also indicated for each curve.

interaction levels with $1 \leqslant n \leqslant 5$. In the field-free limit, $\rho_{e}$ is about $22.5^{\circ}$ for $1 \leqslant n \leqslant 4$ and $15^{\circ}$ for $n=5$, as can be inferred from the free internal rotation functions. When the intensity of the laser beam increases, $\rho_{e}$ displays a complicated behavior except for $n=1$. For the largest intensity value, torsional alignment takes place for the $n=1$ and 4 levels as their $\rho_{e}$-values are close to zero; for the $n=1$ level, this is consistent with Fig. 4. The $n=2,3$, and 5 levels display no torsional alignment as their $\rho_{e}$ values are much larger than zero. For the $n=5$ level, this is expected from the form of its torsional function, given in Fig. 6. Figure 9 displays the same results as described in the legend of Fig. 8, but for Case II potential. In the field-free limit, the torsional function is centered near $\rho=0$. Figure 9 shows indeed that for a zero intensity $\rho_{e}$ is small and is only $3^{\circ}$ for all five levels. When the intensity of the laser beam increases, $\rho_{e}$ decreases slowly down to $2.5^{\circ}$ for $n=1$. For levels with $n>1, \rho_{e}$ displays a more complicated behavior and can be as large as $8^{\circ}$. For $n=2$, the variations of $\rho_{e}$ are consistent with Fig. 7 and a large increase takes place for $I=7.5 \times 10^{13} \mathrm{~W} / \mathrm{cm}^{2}$. Figure 9 emphasizes that torsional alignment takes place for $n=1,4$, and 5 , as for these levels $\rho_{e}$ is close to 0 . Similar qualitative conclusions hold for Case III potential for which torsional alignment only takes place for a few levels. At last, for Case IV potential, $\rho_{e}$ varies slowly with the laser beam intensity for all levels and remains close to $22^{\circ}$.

\section{Thermal averages}

In previous sections the behavior of individual molecules were dealt with. Here we give results for a thermal ensemble. For that, we assume that the ensemble is initially described by a Boltzmann distribution. We further assume that the time evolution is adiabatic regardless of the initial state. This implies an adiabatic transfer of populations from the initial field-free ensemble to the nonzero field ensemble [24].

Thermal averages involving the direction cosines matrix elements between the space-fixed $Z$ axis and the molecular-fixed $x, y$, and $z$ axes, $\Phi_{Z x}, \Phi_{Z y}$, and $\Phi_{Z z}$ were calculated. Figure 10 displays the variations of the thermal averages $\left\langle\left\langle\Phi_{Z x}^{2}\right\rangle\right\rangle$ and $\left\langle\left\langle\Phi_{Z z}^{2}\right\rangle\right\rangle$ as a function of the intensity of the laser beam for

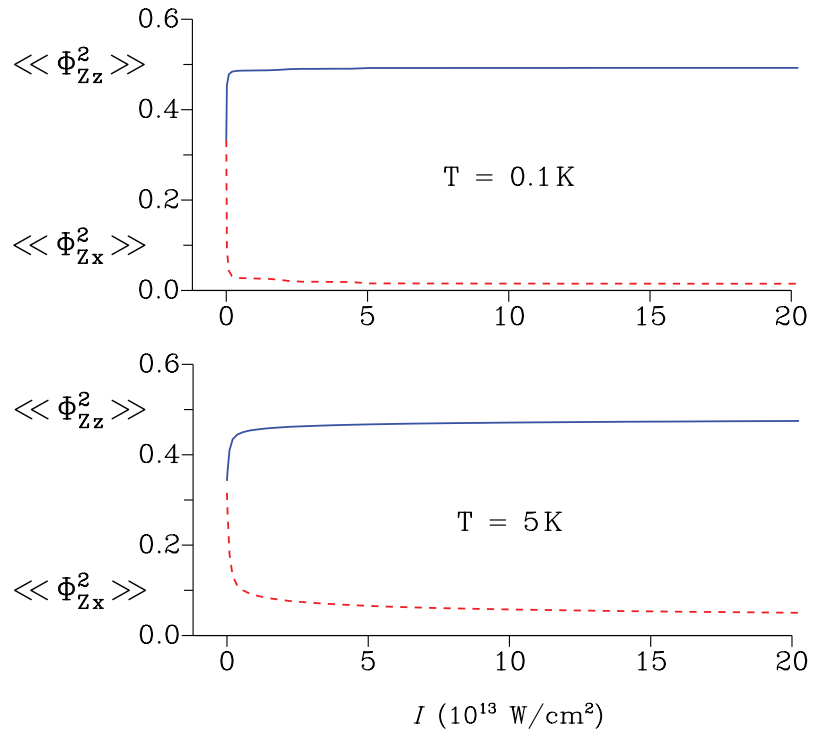

FIG. 10. (Color online) Thermal average of the squared direction cosine $\Phi_{Z x}^{2}$, solid line, and $\Phi_{Z z}^{2}$, dashed line, as a function of the intensity of the laser beam for Case I potential and for temperatures equal to $0.1 \mathrm{~K}$, upper panel, and $5 \mathrm{~K}$, lower panel. The thermal average $\left\langle\left\langle\Phi_{Z y}^{2}\right\rangle\right\rangle$ is not drawn as it is equal to $\left\langle\left\langle\Phi_{Z x}^{2}\right\rangle\right\rangle$.

Case I potential and for two temperatures. The thermal average $\left\langle\left\langle\Phi_{Z y}^{2}\right\rangle\right\rangle$ does not appear in this figure as it is equal to $\left\langle\left\langle\Phi_{Z x}^{2}\right\rangle\right\rangle$ for symmetry reasons. In the field-free limit, as shown by Fig. 10, we have $\left\langle\left\langle\Phi_{Z x}^{2}\right\rangle\right\rangle=\left\langle\left\langle\Phi_{Z y}^{2}\right\rangle\right\rangle=\left\langle\left\langle\Phi_{Z z}^{2}\right\rangle\right\rangle=1 / 3$. When the intensity of the laser beam increases, $\left\langle\left\langle\Phi_{Z x}^{2}\right\rangle\right\rangle=\left\langle\left\langle\Phi_{Z y}^{2}\right\rangle\right\rangle$ rises from $1 / 3$ to $1 / 2$, while $\left\langle\left\langle\Phi_{Z z}^{2}\right\rangle\right\rangle$ decreases from $1 / 3$ to 0 . For the lowest temperature, a fast variation takes place; for the highest one, the variation is slower. In agreement with Eq. (15), this behavior is consistent with the molecule-fixed $z$ axis becoming perpendicular to the space-fixed $Z$ and a rotational function becoming localized near $\theta=\pi / 2$ for a large enough intensity of the laser beam. This angular alignment is similar to that of a symmetric top molecule with a polarizability tensor corresponding to the prolate spheroidal case. For Cases II, III, and IV potentials, the thermal averages of the squared direction cosine matrix elements display qualitatively the same behavior when the intensity of the laser beam increases.

Thermal averages of the angle of internal rotation $\rho_{t}$ were also calculated expressing it in terms of the thermal average of $\cos 4 \rho$ with $\rho_{t}=\frac{1}{4} \cos ^{-1}\langle\langle\cos 4 \rho\rangle\rangle$. The variations of $\rho_{t}$ with the intensity of the laser beam are plotted in Fig. 11 for two temperatures and for Cases I, II, and III potentials. For the lowest temperature, $\rho_{t}$ becomes close to zero when the intensity of the laser beam increases. For Case I and II potentials, this occurs for an intensity as low as $10^{14} \mathrm{~W} / \mathrm{cm}^{2}$. For Case III potential, $\rho_{t}$ is still $10^{\circ}$ for the largest value of the intensity. For the highest temperature, $\rho_{t}$ does not go to zero, even for the largest intensity value. This behavior can be understood more easily with the help of Figs. 8 and 9 . For the lowest temperature, the thermal averages only involve low-lying levels like the $n=1$ levels in these figures, which are characterized by a value of $\rho_{e}$ going to zero with increasing values of the intensity of the laser beam. For the highest temperature, the thermal averages involve higher-lying levels 


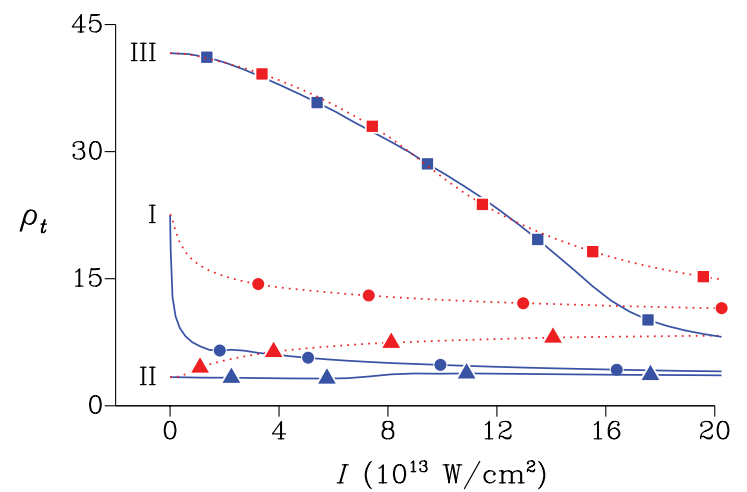

FIG. 11. (Color online) The thermal average $\rho_{t}$ is plotted as a function of the intensity of the laser field for temperatures of $0.1 \mathrm{~K}$, solid line, and $5 \mathrm{~K}$, dotted line. Case I, II, and III potentials are identified using circles, triangles, and squares, respectively.

like the $n>1$ levels in Figs. 8 and 9, which are characterized by a value of $\rho_{e}$ which does not always go to zero. The thermal average $\rho_{t}$ is not plotted in Fig. 11 for Case IV potential because, as can be inferred from the results in Sec. IV B, it barely varies with the intensity of the laser beam and remains close to $22^{\circ}$ for both temperatures.

\section{DISCUSSION}

The present article reports the results of a theoretical investigation into the behavior of a nonrigid molecule subject to a strong electric field. A detailed account of the theory presented in our previous paper [14] is given and additional evidence supporting the conclusions of this paper is presented.

After presenting the exact Hamiltonian, it is shown that a DVR approach $[15,16]$ allows us to calculate rotation-torsionStark energy levels for fixed values of the torsional angle $\rho$. These results are applied to nonrigid biphenyl-like molecules displaying a large amplitude torsional motion corresponding to respective rotations of their two phenyl groups. It is emphasized that for these highly symmetrical molecules, our theoretical approach yields symmetry labels for the energy levels in $G_{16}^{(2)}$, leading to a better understanding of the effects of the external electric field.

Numerical results are obtained in the form of averaged torsional functions as well as expectation values and thermal averages of the torsional angle. Four different hindering potentials characterized by different barrier heights and minima are taken into account. Averaged torsional functions are plotted in Figs. 4 to 7 for an intensity of a circularly polarized laser beam going from 0 to $2 \times 10^{14} \mathrm{~W} / \mathrm{cm}^{2}$. In these $2 \mathrm{D}$ figures, unlike in Ref. [14], the intensity varies smoothly. It is also important to stress that the symmetry labeling using $G_{16}^{(2)}$ allows us to follow adiabatically a given level for all intensities of the laser beam.

Expectation values for the angle of internal rotation are plotted in Figs. 8 and 9. These results are consistent with torsional alignment taking place for levels correlating to low energy values in the field-free limit, as long as the height of the barriers hindering the internal rotation is smaller than $500 \mathrm{~cm}^{-1}$. The corresponding torsional functions become localized near values of $\rho$ corresponding to the four planar configurations that minimize the interaction with the laser field.
For levels correlating to higher energies in the field-free limit, no torsional alignment occurs. This significant result allows us to understand why, as already stressed [14], the torsional alignment is destroyed when the temperature increases.

The results in Figs. 8 and 9 should be compared to those in Fig. 4 of Ref. [12]. The torsional alignment factor plotted in that figure is quite similar to the expectation values considered in the present work. In Fig. 4 of Ref. [12], it can be seen that for all types of torsional potential, the torsional alignment factor increases smoothly with the effective interaction strength. This behavior is similar to that of the $n=1$ level in Figs. 8 and 9 . The complicated behavior found in this work for $n>1$ levels cannot be obtained using the simplified approach of Ref. [12] because in that approach the overall rotation is ignored.

Rotational alignment also plays an important role in torsional alignment. In effect, the present results show that, regardless of the nature of the hindering potential, the angular alignment is similar to that of a symmetric-top molecule with a polarizability tensor corresponding to the prolate spheroidal case. This kind of alignment takes place for laser beams with an intensity as low as $5 \times 10^{13} \mathrm{~W} / \mathrm{cm}^{2}$ and for a temperature as high as $5 \mathrm{~K}$.

The complicated dependence of the averaged torsional function with the laser intensity, displayed in Figs. 6 and 7, is due to the existence of avoided crossings that by hypothesis are transversed adiabatically; that is, we assume that the laser envelope varies slowly enough as to guarantee a truly adiabatic time evolution. Note that the quantum adiabatic theorem holds strictly in the limit corresponding to an infinitely slow timedependent perturbation. For perturbations of finite duration, the validity of an adiabatic approximation depends on, among other things, the existence of avoided crossings between instantaneous energy levels. Thus, if the avoided crossings are weak, the time required to achieve a truly adiabatic evolution could be so large that a practical implementation of such adiabatic strategy would be prevented. Additionally, adiabatic following implies strong changes in the character of the functions before and after encountering an avoided crossing. Thus, maximum overlap between the involved functions is obtained instead by fully diabatic following.

Sophisticated methods have been proposed to control torsional motions. These methods are based on a two-step strategy. In the first step, a long nonresonant elliptically polarized laser pulse gives rise to $3 \mathrm{D}$ alignment. The field must be weak enough for the torsional degree of freedom to be unaffected. In the second step, a short pulse imparts a kick to the molecule, initiating torsion about a stereogenic axis [10]. Experimental evidence was given in Refs. $[9,10]$ that the combined effect of the two lasers improves the torsional alignment. The theoretical model developed by Stapelfeldt and coworkers assumes that the kicking pulse interacts with a prealigned molecule whose torsional eigenstates are essentially field-free torsional eigenfunctions. This view is consistent with our results since the strong interaction between torsion and rotation mediated by the field is avoided for the first adiabatic laser. An interesting issue, related to the design of future experiments involving additional lasers, is whether the 3D alignment achieved by the first adiabatic laser will be conserved or lost when the second laser kicks the molecule and excites the torsion. In effect, the model by Madsen and 
coworkers [8-10] implies that at the time the second laser kicks the molecule the wave functions are essentially given by product functions of the form

$$
\Psi\left(t_{0}\right)=\Phi_{\mathrm{tor}, n}^{\mathrm{ff}} \Phi_{\mathrm{rot}, n}^{p},
$$

where $\Phi_{\mathrm{tor}, n}^{\mathrm{ff}}$ is a field-free torsional eigenstate and $\Phi_{\mathrm{rot}, n}^{p}$ is a $3 \mathrm{D}$-aligned pendular rotational state given by a linear combination of field-free rotational eigenstates. After the kick, this wave function evolves to

$$
\Psi\left(t_{f}\right)=\sum_{n} c_{n} \Phi_{\mathrm{tor}, n}^{\mathrm{ff}} \Phi_{\mathrm{rot}, n}^{p} .
$$

When the second laser ends, and during the further timeevolution driven by the first adiabatic laser that still is on, the molecule remains well aligned as far as each $\Phi_{\text {rot }, n}^{p}$ function entering in the linear combination is well aligned. Basically, the average of Eqs. (14) and (15), with respect to each torsional state changes little with $\Phi_{\mathrm{tor}, n}^{\mathrm{ff}}$. Otherwise, the further evolution under the field due to the first laser will misalign the molecule. A related problem was studied in Ref. [25], where it was shown that a molecule, for which fast intramolecular vibrational relaxation (IVR) takes place, when placed in the presence of an orienting static field, keeps its orientation long after IVR occurs, if the $\mu_{v} / B_{v}$ ratio, where $\mu_{v}$ is the average dipole moment and $B_{v}$ the average rotational constant for vibrational state $v$ is nearly constant for the different states involved in the dynamics.

It has been shown that the coupling between a torsional internal motion and the overall rotation can be strong enough to give rise to rotation-torsion-Stark eigenstates that are delocalized in the torsional angle. Therefore, creation of torsional alignment by adiabatic methods could be impossible to attain. The main conclusion is that prospective strategies aimed to manipulate with lasers large-amplitude molecular vibrations require taking into account the effects coming from other vibrations or from the overall rotation, as is standard practice in molecular spectroscopy [26].

\section{ACKNOWLEDGMENTS}

J.O. acknowledges financial support from the Spanish Government through the MICINN (Project No. FIS201018799).

\section{APPENDIX: WEIGHTS AND POINTS}

For the FBR functions in Eq. (2), the weights and points are the following:

$$
\omega_{\alpha}=\frac{2 \pi}{2 N+1} \text { and } \rho_{\alpha}=\frac{2 \pi(\alpha-N-1)}{2 N+1},
$$

with $1 \leqslant \alpha \leqslant 2 N+1$. The DVR functions take the following expression:

$$
u_{\alpha}(\rho)=g_{N}\left(\rho-\rho_{\alpha}\right) / \sqrt{2 N+1},
$$

where

$$
g_{q}(\rho)=\frac{1}{\sqrt{2 \pi}} \frac{\sin \rho(q+1 / 2)}{\sin \rho / 2} .
$$

For FBR functions $C_{n}(\rho)$, with $0 \leqslant n \leqslant N$, the weights and points are the following:

$$
\omega_{\alpha}=\frac{2 \pi}{N+1} \text { and } \rho_{\alpha}^{c}=\frac{\pi(N-\alpha+3 / 2)}{N+1},
$$

where $1 \leqslant \alpha \leqslant N+1$. For the FBR functions $S_{n}(\rho)$, with $1 \leqslant n \leqslant N$, the weights and points are the following:

$$
\omega_{\alpha}=\frac{2 \pi}{N+1} \quad \text { and } \quad \rho_{\alpha}^{s}=\frac{\pi(N-\alpha+1)}{N+1},
$$

where $1 \leqslant \alpha \leqslant N$
[1] H. Stapelfeldt and T. Seideman, Rev. Mod. Phys. 75, 543 (2003).

[2] J. G. Underwood, B. J. Sussman, and A. Stolow, Phys. Rev. Lett. 94, 143002 (2005).

[3] K. Nakagami, Y. Mizumoto, and Y. Ohtsuki, J. Chem. Phys. 129, 194103 (2008).

[4] B. Friedrich and D. Herschbach, Phys. Rev. Lett. 74, 4623 (1995).

[5] G. R. Kumar, P. Gross, C. P. Safvan, F. A. Rajgara, and D. Mathur, Phys. Rev. A 53, 3098 (1996).

[6] M. Artamonov, T.-S. Ho, and H. Rabitz, Chem. Phys. 328, 147 (2006).

[7] S. Ramakrishna and T. Seideman, Phys. Rev. Lett. 99, 103001 (2007).

[8] C. B. Madsen, L. B. Madsen, S. S. Viftrup, M. P. Johansson, T. B. Poulsen, L. Holmegaard, V. Kumarappan, K. A. Jørgensen, and H. Stapelfeldt, Phys. Rev. Lett. 102, 073007 (2009).

[9] C. B. Madsen, L. B. Madsen, S. S. Viftrup, M. P. Johansson, T. B. Poulsen, L. Holmegaard, V. Kumarappan, K. A. Jørgensen, and H. Stapelfeldt, J. Chem. Phys. 130, 234310 (2009).

[10] J. L. Hansen, J. H. Nielsen, C. B. Madsen, A. T. Lindhardt, M. P. Johansson, T. Skrydstrup, L. B. Madsen, and H. Stapelfeldt, J. Chem. Phys. 136, 204310 (2012).
[11] S. M. Parker, M. A. Ratner, and T. Seideman, J. Chem. Phys. 135, 224301 (2011).

[12] J. Floss, T. Grohmann, M. Leibscher, and T. Seideman, J. Chem. Phys. 136, 084309 (2012).

[13] B. A. Ashwell, S. Ramakrishna, and T. Seideman, J. Chem. Phys. 138, 044310 (2013).

[14] L. H. Coudert, L. F. Pacios, and J. Ortigoso, Phys. Rev. Lett. 107, 113004 (2011).

[15] Z. Bačić and J. C. Light, J. Chem. Phys. 85, 4594 (1986).

[16] J. C. Light and Z. Bačić, J. Chem. Phys. 87, 4008 (1987).

[17] A. J. Merer and J. K. G. Watson, J. Mol. Spectrosc. 47, 499 (1973).

[18] M. A. Mekhtiev, P. D. Godfrey, and V. Szalay, J. Mol. Spectrosc. 180, 42 (1996).

[19] H.-S. Lee and M. E. Tuckerman, J. Phys. Chem. A 110, 5549 (2006).

[20] P. R. Bunker, Molecular Symmetry and Spectroscopy (Academic Press, New York, 1979).

[21] A. R. Edmonds, Angular Momentum in Quantum Mechanics (Princeton University Press, Princeton, 1960). 
[22] E. P. Wigner, Group Theory (Academic Press, New York, 1959).

[23] K. K. Svidzinskii, Theory of the Hyperfine Structure of the Rotational Spectra of Molecules (Soviet Maser Research, D. V. Skobel'tsyn, 1964).
[24] R. Escribano, B. Maté, F. Ortigoso, and J. Ortigoso, Phys. Rev. A 62, 023407 (2000).

[25] J. Ortigoso, G. T. Fraser, and B. H. Pate, Phys. Rev. Lett. 82, 2856 (1999).

[26] J. T. Hougen, J. Mol. Spectrosc. 181, 287 (1997). 